\title{
FROM QUASI-HEREDITARY ALGEBRAS WITH EXACT BOREL SUBALGEBRAS TO DIRECTED BOCSES
}

\author{
TOMASZ BRZEZIŃSKI, STEFFEN KOENIG, AND JULIAN KÜLSHAMMER
}

\begin{abstract}
Up to Morita equivalence, every quasi-hereditary algebra is the dual algebra of a directed bocs or coring. From the bocs, an exact Borel subalgebra is obtained. In this paper a characterisation of exact Borel subalgebras arising in this way is given.
\end{abstract}

\section{INTRODUCTION}

The theorem of Poincaré, Birkhoff and Witt is a fundamental result in the representation theory of semisimple complex Lie algebras $\mathfrak{g}$. When $\mathfrak{b}$ is a Borel subalgebra of $\mathfrak{g}$ and $\mathfrak{h}$ a Cartan subalgebra, then by the PBW theorem induction from $\mathfrak{b}$ to $\mathfrak{g}$ is an exact functor. In particular, inducing up simple $\mathfrak{b}$-modules (which coincide with the simple $\mathfrak{h}$-modules) yields the universal highest weight modules, the Verma modules. The relevant categorical setup is the Bernstein-Gelfand-Gelfand category $\mathcal{O}$, which decomposes into a direct sum of blocks. Each block is equivalent to the module category of a quasi-hereditary algebra, whose standard modules correspond to the Verma modules in the block.

An analogue of the PBW theorem has been established in KKO14 for quasi-hereditary algebras in general, thus also covering Schur algebras of reductive algebraic groups, hereditary algebras and algebras of global dimension two, among many others. More precisely, it has been shown that up to Morita equivalence every quasi-hereditary algebra has an exact Borel subalgebra in the sense of Koen95]. The construction given in KKO14 is based on describing the exact category of standardly filtered modules of a quasi-hereditary algebra as the category of representations of a certain bocs (i.e. a "bimodule over a category with coalgebra structure" [Roi79]) or coring (i.e. a comonoid in the monoidal category of bimodules [Swe75]). This characterises quasi-hereditary algebras. The exact Borel subalgebras obtained by this construction have strong additional properties, which are not present in other such algebras constructed in particular situations such as for category $\mathcal{O}$ (in [Koen95]). In particular, induction preserves significant parts of cohomology and Ringel duality becomes a construction on the directed bocs.

The aim of this article is to analyse and clarify the stronger properties of the exact Borel subalgebras arising from the construction in [KKO14], and to show that these properties are present exactly when the quasi-hereditary algebra $\Lambda$ and the given exact Borel subalgebra $B$ correspond to a directed bocs as in [KKO14]. More precisely, to the ring extension $B \subseteq \Lambda$ we associate the $B$-coring $\operatorname{Hom}_{B \text { op }}(\Lambda, B)$ and translate properties of this ring extension into properties of the corresponding coring. Subsequently we show that directed

Date: July 31, 2019. 
bocses necessarily correspond in this way to quasi-hereditary algebras with exact Borel subalgebras. The first step, Theorem 2.5, translates properties such as $\Lambda$ being a progenerator as right $B$-module into surjectivity of the counit of the $B$-coring $\operatorname{Hom}_{B^{\text {op }}}(\Lambda, B)$ and the existence of a group-like element. The second step, Theorem 2.13, relates invariance of cohomology under induction with the kernel of the counit being projectivising. The main result, Theorem 3.13, then gives the desired converse of the main result of [KKO14, showing that the strong properties of the exact Borel subalgebra $B$ imply that $B \subseteq \Lambda$ comes from a bocs as in [KKO14].

Notation. For a finite dimensional algebra $B$, the category of left $B$-modules is denoted by $\bmod B$, the category of right $B$-modules by $\bmod B^{\mathrm{op}}$. A complete set of representatives of the isomorphism classes of simple left $B$-modules is denoted by $L_{B}(1), \ldots, L_{B}(\mathrm{n})$. The projective cover and injective hull of $L_{B}(\mathrm{i})$ are $P_{B}(\mathrm{i})$ and $I_{B}(\mathrm{i})$, respectively.

\section{RingS AND CORINGS}

In this section we recall the necessary terminology for bocses and corings and describe how certain properties of ring extensions translate to the language of corings by taking the dual.

Definition 2.1. Let $B$ be an algebra. A $B$-coring is a $B$ - $B$-bimodule $W$ with a $B$ $B$-bilinear coassociative comultiplication $\mu: W \rightarrow W \otimes_{B} W$ and a $B$-B-bilinear counit $\varepsilon: W \rightarrow B$, i.e. the following diagrams commute:

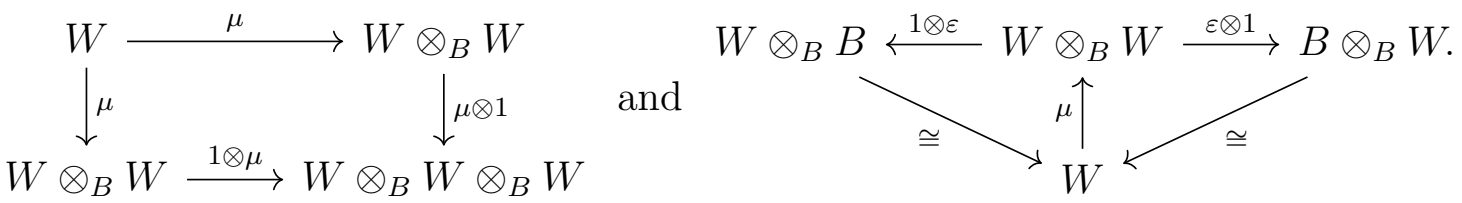

The pair $(B, W)$ is called a bocs..$^{1}$

The reader is referred to [BW03] and [BSZ09] for comprehensive treatments of corings and bocses. Bocses will arise in this article as duals of ring extensions. The following proposition is well-known. A slightly more general statement can e.g. be found in [Kle84, Theorem 1].

Proposition 2.2. Let $B \subseteq \Lambda$ be a subring of a ring $\Lambda$ such that $\Lambda$ is finitely generated projective as a right $B$-module. Let $W:=\operatorname{Hom}_{B^{\mathrm{op}}}(\Lambda, B)$, and let

$$
\psi: W \otimes_{B} W \longrightarrow \operatorname{Hom}_{B^{\mathrm{op}}}\left(\Lambda \otimes_{B} \Lambda, B\right)
$$

be the map given by $f \otimes g \mapsto f \otimes g$. Then, the map $\psi$ is bijective and $W$ is finitely generated projective, when considered as a left B-module.

\footnotetext{
${ }^{1}$ Although it might not be a generally accepted practice, in order to stay aligned with the existing literature both on representation theory and on Hopf algebras, we use both terms "coring" and "bocs" in this text; coring refers to a bimodule (with a coassociative and counital comultiplication) over a fixed algebra while bocs refers to a pair: algebra, bimodule (with a coassociative and counital comultiplication).
} 
Furthermore, $W$ has the structure of a B-coring with counit given by the map

$$
\varepsilon: \operatorname{Hom}_{B^{\mathrm{op}}}(\Lambda, B) \longrightarrow B, \quad f \longmapsto f(1),
$$

and comultiplication given by the unique map $\mu: W \rightarrow W \otimes_{B} W$ rendering commutative the following diagram:

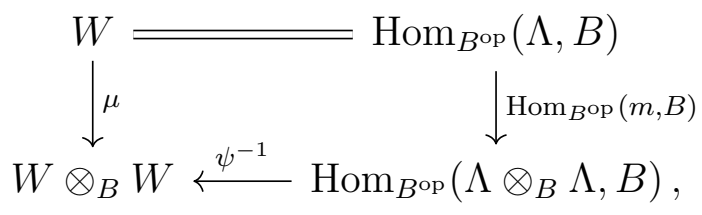

where $m$ is the multiplication map $\Lambda \otimes_{B} \Lambda \rightarrow \Lambda$.

In the theory of corings and bocses, two basic properties are that the counit is surjective, and that the bocs is normal:

Definition 2.3. A bocs $(B, W)$ is called normal if there exists a group-like element in the $B$-coring $W$, i.e. $\omega \in W$ such that $\mu(\omega)=\omega \otimes \omega$ and $\varepsilon(\omega)=1$.

Remark 2.4. - It is easy to see that normality implies that the counit is surjective. The converse is not true in general.

- If $B$ has no (left or right) zero divisors and $\omega \neq 0$, then $\varepsilon(\omega)=1$ in fact follows from $\mu(\omega)=\omega \otimes \omega$, since the counitality implies that $\varepsilon(\omega)$ is an idempotent in $B$.

Our first result answers the question of when a ring extension gives rise to a bocs satisfying these special properties.

Theorem 2.5. Let $B \subseteq \Lambda$ be a ring extension. Assume that $\Lambda$ is finitely generated and projective as a right $B$-module.

(i) The following statements are equivalent:

(1) The inclusion $\iota: B \hookrightarrow \Lambda$ splits as a map of right $B$-modules.

(2) $\Lambda / \iota(B)$ is projective as a right $B$-module.

(3) $\Lambda$ is a projective generator as a right $B$-module.

(4) The counit of the right dual coring $\operatorname{Hom}_{B^{\mathrm{op}}}(\Lambda, B)$ is surjective.

(ii) The following statements are equivalent:

(1) There is a splitting of the inclusion $\iota: B \hookrightarrow \Lambda$ as right $B$-modules whose kernel is a right ideal of $\Lambda$.

(2) The right dual coring $\operatorname{Hom}_{B^{\mathrm{op}}}(\Lambda, B)$ is normal.

Proof. (i) We start by showing that (1) and (2) are equivalent. Assume that (1) holds, i.e. that the exact sequence $0 \rightarrow B \stackrel{\iota}{\rightarrow} \Lambda \rightarrow \Lambda / \iota(B) \rightarrow 0$ splits. In particular, $\Lambda / \iota(B)$ is a direct summand of the projective right $B$-module $\Lambda$ and thus is itself projective. Therefore (2) holds. Conversely, the projectivity of $\Lambda / \iota(B)$ implies that the sequence splits in the category of right $B$-modules.

That (1) implies (3) follows from the fact that $\Lambda$ is a generator as a right $B$-module, since $B$ is a direct summand of $\Lambda$. 
The direction that (3) implies (4) uses parts of Morita theory. Recall that an equivalent formulation of $\Lambda$ being a generator over $B$ is that the trace of $\Lambda$ in $B$ is $B$, i.e. that $B=\sum_{g \in \operatorname{Hom}_{B} \text { op }(\Lambda, B)} g(\Lambda)$, see e.g. [Lam99, Theorem 18.8]. In particular, for every $b \in B$, there exist $a_{i} \in \Lambda$ and $g_{i} \in \operatorname{Hom}_{B^{\text {op }}}(\Lambda, B)$ such that

$$
b=\sum_{i} g_{i}\left(a_{i}\right)=\sum_{i} g_{i}\left(\lambda_{a_{i}}(1)\right)
$$

where $\lambda_{a_{i}}$ denotes the right $B$-module endomorphism of $\Lambda$ given by left multiplication with $a_{i}$. In particular, $b=\varepsilon\left(\sum_{i} g_{i} \circ \lambda_{a_{i}}\right)$, and therefore the counit is surjective.

For the remaining direction (4) implies (1) observe that $\varepsilon$ is surjective if and only if $\operatorname{Hom}_{B^{\mathrm{op}}}(\iota, B)$ is surjective as the following diagram commutes

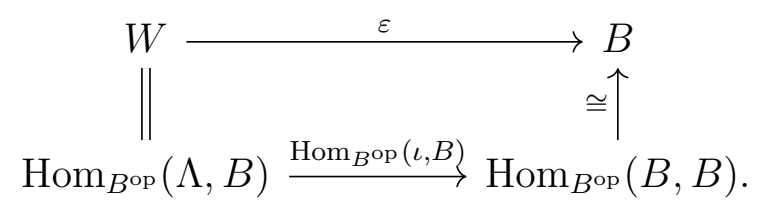

Consider the exact sequence of right $B$-modules $0 \rightarrow B \stackrel{\iota}{\rightarrow} \Lambda \rightarrow \Lambda / \iota(B) \rightarrow 0$. Applying $\operatorname{Hom}_{B^{\text {op }}}(-, B)$ yields the long exact sequence

$$
\begin{aligned}
0 \longrightarrow \operatorname{Hom}_{B^{\mathrm{op}}}(\Lambda / \iota(B), B) \longrightarrow & \operatorname{Hom}_{B^{\mathrm{op}}}(\Lambda, B) \stackrel{\operatorname{Hom}_{B^{\mathrm{op}}}(\iota, B)}{\longrightarrow} \operatorname{Hom}_{B^{\mathrm{op}}}(B, B) \\
& \longrightarrow \operatorname{Ext}_{B^{\mathrm{op}}}^{1}(\Lambda / \iota(B), B) \rightarrow \operatorname{Ext}_{B^{\mathrm{op}}}^{1}(\Lambda, B) .
\end{aligned}
$$

The last term of this sequence vanishes as $\Lambda$ is projective as a right $B$-module. Moreover, $\operatorname{Hom}_{B^{\text {op }}}(\iota, B)$ is surjective. Therefore, $\operatorname{Ext}_{B^{\text {op }}}^{1}(\Lambda / \iota(B), B)=0$ and the exact sequence of right $B$-modules $0 \rightarrow B \stackrel{\iota}{\rightarrow} \Lambda \rightarrow \Lambda / \iota(B) \rightarrow 0$ splits.

(ii) This is proved in [Kle84, Theorem 3].

Example 2.6. For illustration, we provide non-instances of the preceding theorem.

(i) Let $\Lambda=M_{2}(\mathbb{k})$ and let $B$ be the subalgebra of upper triangular matrices. Let $e_{2}=\left(\begin{array}{ll}1 & 0 \\ 0 & 0\end{array}\right) \in B$. Then

$$
\Lambda_{B}=\left(\begin{array}{ll}
1 & 0 \\
0 & 0
\end{array}\right) B \oplus\left(\begin{array}{ll}
0 & 0 \\
1 & 0
\end{array}\right) B \cong\left(e_{2} B\right)^{\oplus 2}
$$

is finitely generated and projective as a right $B$-module but not a projective generator. Thus, the counit of the corresponding coring is not surjective.

(ii) Let $\Lambda=M_{2}(\mathbb{k})$ and let $B$ be the subalgebra of diagonal matrices. In this case the algebra $\Lambda$ is a projective generator over $B$. However, the corresponding coring (described in [Roi79, p. 308]) is not normal.

Continuing the discussion on how to translate properties of bocses to properties of ring extensions we consider the property that the kernel of the counit $\varepsilon: W \rightarrow B$ is a projective bimodule (and the slightly weaker property of being projectivising in the sense of [BB91]). We slightly altered the terminology in [BB91] by having a separate terminology for the two properties. 
Definition 2.7. Let $B$ be a ring. A $B$-B-bimodule $U$ is called left projectivising if $U \otimes_{B} X$ is projective as a left $B$-module for each $X \in \bmod B$. Symmetrically, a bimodule is called right projectivising if $X \otimes_{B} U$ is projective as a right $B$-module for each $X \in$ $\bmod B^{\mathrm{op}}$. A bimodule $U$ is called projectivising if it is both left and right projectivising.

Any $B$ - $B$-bimodule of the form $\bigoplus_{i, j} P_{i} \otimes_{\mathbb{k}} Q_{j}$ for a projective left module $P_{i}$ and a projective right module $Q_{j}$ is both left and right projectivising. Over a perfect field, a $B$ $B$-bimodule is projective if and only if it is isomorphic to a direct summand of a module of the above form. In particular, it is left and right projectivising. In this case, the following proposition shows that also the converse holds.

Proposition 2.8 (AR91, Theorem 3.1]). Let $B$ be a finite dimensional algebra over a perfect field $\mathbb{k}$ and let $W$ be a $B$-B-bimodule such that $W$ is projective as a right module and $W \otimes_{B}(B / \operatorname{rad}(B))$ is projective as a left module. Then, $W$ is projective as a $B$ - $B$ bimodule. In particular, a right projective and left projectivising bimodule is projective as a bimodule.

The next statement follows from the proof of [BB91, Lemma 3.6]. For convenience of the reader we include the proof.

Lemma 2.9. Let $B$ be a ring. $A B$-B-bimodule $U$ that is projective as a right $B$-module is left projectivising if and only if $\operatorname{Hom}_{B}(U, X)$ is injective for every left $B$-module $X$.

Proof. Let $0 \rightarrow M_{1} \rightarrow M_{2} \rightarrow M_{3} \rightarrow 0$ be an exact sequence of left $B$-modules. Since $U$ is projective as a right $B$-module, also the sequence

$$
0 \rightarrow U \otimes_{B} M_{1} \rightarrow U \otimes_{B} M_{2} \rightarrow U \otimes_{B} M_{3} \rightarrow 0
$$

is exact. Let $X$ be a left $B$-module. Applying the functor $\operatorname{Hom}_{B}(-, X)$ to the above exact sequence yields the exact sequence

$$
\begin{aligned}
0 \longrightarrow \operatorname{Hom}_{B}\left(U \otimes_{B} M_{3}, X\right) & \longrightarrow \operatorname{Hom}_{B}\left(U \otimes_{B} M_{2}, X\right) \\
& \longrightarrow \operatorname{Hom}_{B}\left(U \otimes_{B} M_{1}, X\right) \longrightarrow \operatorname{Ext}_{B}^{1}\left(U \otimes_{B} M_{3}, X\right) .
\end{aligned}
$$

Similarly, applying $\operatorname{Hom}_{B}\left(-, \operatorname{Hom}_{B}(U, X)\right)$ to the given exact sequence yields the exact sequence

$$
\begin{aligned}
0 \rightarrow \operatorname{Hom}_{B}\left(M_{3}, \operatorname{Hom}_{B}(U, X)\right) & \rightarrow \operatorname{Hom}_{B}\left(M_{2}, \operatorname{Hom}_{B}(U, X)\right) \\
& \rightarrow \operatorname{Hom}_{B}\left(M_{1}, \operatorname{Hom}_{B}(U, X)\right) \rightarrow \operatorname{Ext}_{B}^{1}\left(M_{3}, \operatorname{Hom}_{B}(U, X)\right) .
\end{aligned}
$$

Adjointness of tensor and Hom provides natural identifications of the three Hom-spaces in the exact sequence 2.9 .2 with those in the exact sequence 2.9 .3$)$. When $U$ is left projectivising, each sequence (2.9.1) and hence also (2.9.2) is split exact. Therefore the corresponding part of the sequence (2.9.3) is split exact, too, and $\operatorname{Hom}_{B}(U, X)$ is injective for every left $B$-module $X$. Conversely, injectivity of $\operatorname{Hom}_{B}(U, X)$ shows that (2.9.3) starts with a split exact sequence and thus $U$ is left projectivising. 
Before stating which property of a subalgebra corresponds to the kernel of the counit of the dual coring being projectivising we recall the notion of a right algebra from [BB91, $\left.\left(1.1^{\prime}\right)\right]$.

Definition 2.10. Let $(B, W)$ be a bocs. The right algebra $R$ of $W$ is the algebra $\operatorname{Hom}_{B}(W, B)^{\mathrm{op}}$. Here, the product of $s, t \in \operatorname{Hom}_{B}(W, B)$ is given as the composition of the following maps

$$
W \stackrel{\mu}{\rightarrow} W \otimes_{B} W \stackrel{1 \otimes s}{\longrightarrow} W \otimes_{B} B \stackrel{\cong}{\rightarrow} W \stackrel{t}{\rightarrow} B,
$$

and the identity is $\varepsilon$.

Remark 2.11. As mentioned, the terminology is from the theory of bocses. The literature on corings calls this algebra the opposite algebra of the left dual algebra, see e.g. [BW03]. That $\operatorname{Hom}_{B}(W, B)$ is an algebra for any $B$-coring $W$ as in Definition 2.10 was observed by Sweedler already in [Swe75, 3.2 Proposition]. The corresponding structure on $\operatorname{Hom}_{B^{\mathrm{op}}}(W, B)$ is called the left algebra in the bocs literature or the right dual algebra in the coring literature.

The following result from [BB91, (3.8)] translates the property of a bocs to have a surjective counit to the language of ring extensions.

Lemma 2.12. Let $(B, W)$ be a bocs with surjective counit. Let $\bar{W}:=\operatorname{ker} \varepsilon$ be left projectivising and right projective. Let $R$ be its right algebra. Then, the homomorphisms $\operatorname{Ext}_{B}^{i}(M, N) \rightarrow \operatorname{Ext}_{R}^{i}\left(R \otimes_{B} M, R \otimes_{B} N\right)$ induced by the induction functor are epimorphisms for $i=1$ and isomorphisms for $i \geq 2$.

The main result of this section is the converse to Lemma 2.12. The proofs of the two results follow the same lines.

Theorem 2.13. Let $B \subseteq \Lambda$ be a subalgebra such that $\Lambda$ is a projective generator for $B$ as a right $B$-module. Assume furthermore, that the homomorphisms

$$
\operatorname{Ext}_{B}^{i}(M, N) \rightarrow \operatorname{Ext}_{\Lambda}^{i}\left(\Lambda \otimes_{B} M, \Lambda \otimes_{B} N\right)
$$

induced by the induction functor are epimorphisms for $i \geq 1$ and isomorphisms for $i \geq 2$. Let $W=\operatorname{Hom}_{B^{\mathrm{op}}}(\Lambda, B)$ be the right dual coring for $\Lambda$ and define $\bar{W}:=\operatorname{ker} \varepsilon$. Then $\bar{W}$ is left projectivising.

Proof. By Theorem 2.5, $W=\operatorname{Hom}_{B^{\mathrm{op}}}(\Lambda, B)$ is a $B$-coring with surjective counit. Hence, there is a short exact sequence of $B$ - $B$-bimodules

$$
0 \longrightarrow \bar{W} \longrightarrow W \longrightarrow B \longrightarrow 0
$$

which splits as a sequence of left modules. Applying $\operatorname{Hom}_{B}(-, N)$ for a left $B$-module $N$ thus gives a short exact sequence

$$
0 \longrightarrow N \longrightarrow \operatorname{Hom}_{B}(W, N) \longrightarrow \operatorname{Hom}_{B}(\bar{W}, N) \longrightarrow 0 .
$$

As $\Lambda$ is finitely generated projective as a right $B$-module, there is an isomorphism

$$
\operatorname{Hom}_{B}(W, N) \cong \Lambda \otimes_{B} N .
$$


Furthermore, by the Eckmann-Shapiro Lemma, see e.g. [BB91, (3.1)], the same assumption yields isomorphisms

$$
\operatorname{Ext}_{B}^{i}\left(M, \Lambda \otimes_{B} N\right) \cong \operatorname{Ext}_{\Lambda}^{i}\left(\Lambda \otimes_{B} M, \Lambda \otimes_{B} N\right),
$$

for all left $B$-modules $M, N$. Applying $\operatorname{Hom}_{B}(M,-)$ to the exact sequence (2.13.1), using these isomorphisms and denoting $\operatorname{Hom}_{B}(\bar{W}, N)$ by $I$ yields the long exact sequence

$$
\cdots \longrightarrow \operatorname{Ext}_{B}^{i}(M, N) \longrightarrow \operatorname{Ext}_{\Lambda}^{i}\left(\Lambda \otimes_{B} M, \Lambda \otimes_{B} N\right) \longrightarrow \operatorname{Ext}_{B}^{i}(M, I) \longrightarrow \ldots
$$

The assumed epimorphism for $i=1$ and isomorphisms for $i \geq 2$ then imply $\operatorname{Ext}_{B}^{i}(M, I)=$ 0 , for all $i \geq 1$. Hence, $I=\operatorname{Hom}_{B}(\bar{W}, N)$ is an injective module for all left $B$-modules $N$. By Lemma 2.9, this is equivalent to ker $\varepsilon$ being left projectivising.

We finish this section by providing an example of a non-instance of the preceding theorem from [KKO14, Appendix A.4].

Example 2.14. Let $\Lambda$ be the path algebra of the quiver

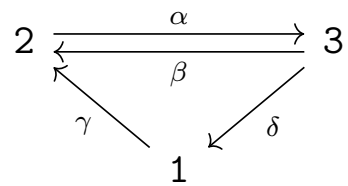

with relations $\gamma \delta=0=\alpha \beta$. Then $\Lambda$ has a subalgebra $B$ given by the subquiver with arrows $\alpha$ and $\gamma$, over which it is a projective generator as a right $B$-module. Then $0=\operatorname{Ext}_{B}^{1}(L(1), L(3))$ but $\operatorname{dim} \operatorname{Ext}_{B}^{1}\left(\Lambda \otimes_{B} L(1), \Lambda \otimes_{B} L(3)\right)=1$.

\section{Homological exact Borel subalgebras}

In this section the results obtained in the previous section are applied to the setting of quasi-hereditary algebras and exact Borel subalgebras. For further reading on quasihereditary algebras we suggest the original articles [Sc087, CPS88] and the survey articles [DR92, KK99]. We start by recalling these notions. Throughout this section assume that $\mathbb{k}$ is a splitting field for $\Lambda$.

Definition 3.1. A finite dimensional algebra $\Lambda$ is quasi-hereditary if there exist modules $\Delta(\mathrm{i}), \mathrm{i} \in\{1, \ldots, \mathrm{n}\}$ satisfying

$(\mathrm{QH} 1) \operatorname{End}_{\Lambda}(\Delta(\mathrm{i})) \cong \mathbb{k}$.

(QH2) $\operatorname{Hom}_{\Lambda}(\Delta(\mathrm{i}), \Delta(\mathrm{j})) \neq 0 \Rightarrow \mathrm{i} \leq \mathrm{j}$.

(QH3) $\operatorname{Ext}_{\Lambda}^{1}(\Delta(\mathrm{i}), \Delta(\mathrm{j})) \neq 0 \Rightarrow \mathrm{i}<\mathrm{j}$.

(QH4) ${ }_{\Lambda} \Lambda \in \mathcal{F}(\Delta)$, where $\mathcal{F}(\Delta)$ denotes the subcategory of all $\Lambda$-modules which can be filtered by the $\Delta(i)$, i.e.

$$
\mathcal{F}(\Delta)=\left\{M \in \bmod \Lambda \mid \exists 0=M_{0} \subseteq M_{1} \subseteq \cdots \subseteq M_{s} \text { such that } \forall t \exists i_{t}: M_{t} / M_{t+1} \cong \Delta\left(i_{t}\right)\right\} .
$$

The modules $\Delta(i)$ are called the standard modules. 
Example 3.2. Examples of quasi-hereditary algebras include blocks of the BernsteinGelfand-Gelfand category $\mathcal{O}$, classical and quantised Schur algebras of reductive algebraic groups and of symmetric groups and of Brauer algebras, and algebras of global dimension smaller than or equal to two. For later use, we give an example of a monomial quasihereditary algebra. It appeared in a previous version of [GS17]: Let $\Lambda$ be the path algebra of the quiver

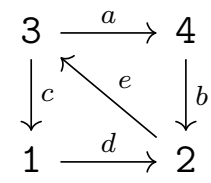

modulo the relations $d c-b a, a e, e b$. Its standard modules are given by $\Delta(1)=L(1)$, $\Delta(2)=L(2), \Delta(3)=\begin{aligned} & 3 \\ & 1\end{aligned}$, and $\Delta(4)=\frac{4}{2}$. For further examples, see e.g. [DR92, KK99].

Remark 3.3. (i) Being quasi-hereditary can also be defined using a dual condition: An algebra is quasi-hereditary if and only if there exist modules $\nabla(i), i \in\{1, \ldots, n\}$ satisfying

$\left(\mathrm{QH1} 1^{\prime}\right) \operatorname{End}_{\Lambda}(\nabla(\mathrm{i})) \cong \mathbb{k}$.

$\left(\mathrm{QH} 2^{\prime}\right) \operatorname{Hom}_{\Lambda}(\nabla(\mathrm{i}), \nabla(\mathrm{j})) \neq 0 \Rightarrow \mathrm{i} \geq \mathrm{j}$.

(QH3') $\operatorname{Ext}_{\Lambda}^{1}(\nabla(i), \nabla(j)) \neq 0 \Rightarrow i>j$.

$\left(\mathrm{QH} 4{ }^{\prime}\right) D \Lambda \in \mathcal{F}(\nabla)$.

(ii) The $\Delta(i)$ and $\nabla(i)$ are uniquely determined (given the implicit order on the simple modules). They can be defined as

$$
\Delta(\mathrm{i})=\frac{P(\mathrm{i})}{\sum_{\substack{P(\mathbf{j}) \rightarrow P(\mathrm{i}) \\ \mathrm{j}>\mathrm{i}}} \operatorname{Im} g}
$$

and $\nabla(i)$ is the maximal submodule of $I(i)$ all of whose composition factors are of the form $L(j)$ for $j \leq i$. To check that an algebra is quasi-hereditary, one can also check that (QH1) and (QH4) (or (QH1') and (QH4')) are satisfied for these explicitly defined modules.

We recall the notion of an exact Borel subalgebra from [Koen95] and provide the new notion of a homological exact Borel subalgebra which will be precisely the class of exact Borel subalgebras giving rise to directed corings.

Definition 3.4. Let $\Lambda$ be a quasi-hereditary algebra. A subalgebra $B \subseteq \Lambda$ is called an exact Borel subalgebra if its isomorphism classes of simple modules can be indexed by the same indexing set $\{1, \ldots, \mathrm{n}\}$ as the standard modules for $\Lambda$ and

(B1) the algebra $\Lambda$ is directed, i.e. it is quasi-hereditary with simple standard modules,

(B2) the algebra $\Lambda$ is projective when considered as a right $B$-module,

(B3) the standard modules for $\Lambda$ can be obtained as $\Delta_{\Lambda}(\mathrm{i})=\Lambda \otimes_{B} L_{B}(\mathrm{i})$. 
(H) If the subalgebra $B$ furthermore has the property that the homomorphisms

$$
\operatorname{Ext}_{B}^{i}(M, N) \longrightarrow \operatorname{Ext}_{\Lambda}^{i}\left(\Lambda \otimes_{B} M, \Lambda \otimes_{B} N\right)
$$

induced by the induction functor are epimorphisms for $i \geq 1$ and isomorphisms for $i \geq 2$, then $B$ is called a homological exact Borel subalgebra.

(N) An exact Borel subalgebra is said to be normal if there is a splitting of the inclusion $\iota: B \hookrightarrow \Lambda$ as right $B$-modules whose kernel is a right ideal of $\Lambda$.

(R) A normal exact Borel subalgebra is said to be regular if there are isomorphisms $\operatorname{Ext}_{B}^{i}\left(L_{B}(\mathrm{j}), L_{B}(\mathrm{k})\right) \longrightarrow \operatorname{Ext}_{\Lambda}^{i}\left(\Lambda \otimes_{B} L_{B}(\mathrm{j}), \Lambda \otimes_{B} L_{B}(\mathrm{k})\right)$ for all $i \geq 1$ and all $\mathrm{j}, \mathrm{k}$ induced by the induction functor.

Remark 3.5. Note that for a normal exact Borel subalgebra being regular implies being homological as can be seen from the long exact sequence of Ext-groups.

The main result of [KKO14] proves the existence of a regular homological exact Borel subalgebra of every quasi-hereditary algebra up to Morita equivalence. The following example gives one particular instance of this result:

Example 3.6. For the algebra in Example 3.2 the method given in KKO14] produces an exact Borel subalgebra of $\operatorname{End}(\Lambda \oplus P(4))$ which is isomorphic to the path algebra of the quiver

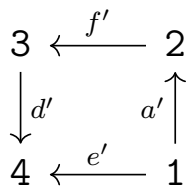

with relation $d^{\prime} f^{\prime}$. More examples of exact Borel subalgebras can be found in KKoen95, KKO14, BK18.

The following lemma is essential for applying Theorem 2.5 to deduce that the counit of the dual coring is surjective.

Lemma 3.7. Let $\Lambda$ be a quasi-hereditary algebra with exact Borel subalgebra B. Then $\Lambda$ is a projective generator for $B$ as a right $B$-module.

Proof. By [Koen95, Theorem A], for an exact Borel subalgebra of a quasi-hereditary algebra there is an isomorphism of $B$-modules $\nabla_{B}(i) \cong \nabla_{\Lambda}(i)$. By the dual definition of quasihereditary using costandard modules, see Remark 3.3, $D \Lambda$ is filtered by $\nabla_{\Lambda}(i)$ with all $i$ appearing at least once. Since $B$ is a quasi-hereditary algebra with simple standard modules, the costandard modules $\nabla_{B}(i)$ are injective. Therefore the filtration of $D \Lambda$ by costandard modules as a left $B$-module splits and $D \Lambda$ is an injective cogenerator as a left $B$-module as all $\nabla_{B}(\mathbf{i})$ appear at least once in a direct sum decomposition. Applying duality, it follows that $\Lambda$ is a projective generator as a right $B$-module.

We now recall the definition of a directed bocs and the main result of [KKO14]. 
Definition 3.8. Let $\mathbb{k}$ be an algebraically closed field. A bocs $(B, W)$ is said to be directed if $B$ is directed, $\bar{W}=$ ker $\varepsilon$ is a projective bimodule and every indecomposable direct summand $B e_{\mathrm{j}} \otimes_{\mathrm{k}} e_{\mathrm{i}} B$ of $\bar{W}$ satisfies $\mathrm{i}<\mathrm{j}$.

Theorem 3.9. Let $\mathbb{k}$ be an algebraically closed field. An algebra $\Lambda$ is quasi-hereditary if and only if it is Morita equivalent to the right algebra $R$ of a directed bocs $(B, W)$. In this case, $B$ is an exact Borel subalgebra of $R$.

In [KKO14] it moreover has been shown that then the category of representations of $(B, W)$ is equivalent as an exact category to the category $\mathcal{F}(\Delta)$ of $\Lambda$-modules with standard filtrations. This description has been used by Bautista, Pérez and Salmerón in [BPS17] to establish the tame-wild dichotomy for the categories $\mathcal{F}(\Delta)$.

Before proving our main result, we recall the notion of regularity for normal bocses which is part of the reduction algorithm used in the proof of Drozd's tame-wild dichotomy theorem. It is essentially due to Kleiner and Roiter [KR77], for the precise formulation see [KM19, Proposition 3.11].

Proposition 3.10. Let $\mathfrak{B}=(B, W)$ be a normal bocs with group-like $\omega$ and projective kernel $\bar{W}$. Define $\partial_{0}: B \rightarrow W$, by $\partial_{0}(a)=\omega a-a \omega$, and assume that there exists $a \in B$ such that $\partial_{0}(a)=\lambda \psi+\sum_{i} c_{i} \psi_{i} b_{i}$ with $b_{i}, c_{i} \in B$ and $\psi \neq \psi_{i}$ are generators of $\bar{W}$ and $0 \neq \lambda \in \mathbb{k}$. Then, there is a bocs $\tilde{B}=(\tilde{B}, \tilde{W})$ with $\tilde{B}=B /(a)$ and $\tilde{W}=\tilde{B} \otimes_{B} W /(\psi) \otimes_{B} \tilde{B}$ such that the following statements hold:

(i) There is an equivalence of categories $\bmod \mathfrak{B} \cong \bmod \tilde{\mathfrak{B}}$.

(ii) If $\mathfrak{B}=(B, W)$ is directed, then $\tilde{\mathfrak{B}}=(\tilde{B}, \tilde{W})$ is directed.

(iii) The right algebra of $\mathfrak{B}$ is Morita equivalent to the right algebra of $\tilde{\mathfrak{B}}$.

Definition 3.11. A bocs $\mathfrak{B}$ is said to be regular if Proposition 3.10 cannot be applied anymore, that is, no $a$ exists with the required property.

The following result was stated by Ovsienko in unpublished notes. A proof can be found in [KM19, Lemma 5.3].

Lemma 3.12. Let $\mathfrak{B}=(B, W)$ be a directed normal bocs with right algebra $R$. Then the following conditions are equivalent:

(1) $\mathfrak{B}$ is regular.

(2) $\operatorname{Ext}_{B}^{1}(\mathbb{L}, \mathbb{L}) \rightarrow \operatorname{Ext}_{R}^{1}(\Delta, \Delta)$ is an isomorphism.

Here, $\mathbb{L}$ is a complete sum of representatives of simple $B$-modules and $\Delta$ is a complete sum of representatives of standard $R$-modules.

We are now ready to prove the main result of this article. 
Theorem 3.13. Let $\mathbb{k}$ be an algebraically closed field. Then there is a one-to-one correspondence:

\{quasi-hereditary algebras with (normal) homological exact Borel subalgebra\}

$$
\begin{gathered}
\uparrow_{1-1} \\
\text { \{directed (normal) bocses\}, }
\end{gathered}
$$

which restricts to a one-to-one correspondence:

$\{$ quasi-hereditary algebras with regular homological exact Borel subalgebra\}

$$
\uparrow 1-1
$$

\{regular directed bocses\}.

Proof. The upwards map is given by sending a directed bocs to its right algebra $R=$ $\operatorname{Hom}_{B}(W, B)^{\mathrm{op}}$. That it is well-defined was proved in [KKO14. For the map in the other direction note that by Lemma 3.7 the algebra $\Lambda$ is a projective generator for $B$. Thus, $\operatorname{Hom}_{B^{\mathrm{op}}}(\Lambda, B)$ is a $B$-coring with surjective counit. Furthermore, Theorem 2.13 shows that $\bar{W}:=\operatorname{ker} \varepsilon$ is projectivising. Since the ground field is assumed to be algebraically closed, in particular perfect, Proposition 2.8 implies that $\bar{W}$ is a projective bimodule and hence is a direct sum of bimodules of the form $B e_{\mathrm{i}} \otimes_{\mathbb{k}} e_{\mathrm{j}} B$. By the definition of exact Borel subalgebra, $B$ is directed. It remains to prove that, for each summand $B e_{\mathbf{i}} \otimes_{\mathbb{k}} e_{\mathbf{j}} B$ of $\operatorname{Hom}_{B^{\mathrm{op}}}(\Lambda, B)$, the inequality $i>j$ holds. Suppose that this is not the case, i.e. $i \leq j$. Note that $\operatorname{Hom}_{\mathbb{k}}\left(L_{B}(j), L_{B}(\mathrm{i})\right) \cong L_{B \otimes_{\mathrm{k}} B \text { op }}((\mathrm{j}, \mathrm{i}))$, the simple module corresponding to the indecomposable projective bimodule $B e_{\mathrm{j}} \otimes_{\mathbb{k}} e_{\mathrm{i}} B$. Applying $\operatorname{Hom}_{B \otimes_{\mathbb{k}} B^{\text {op }}}\left(-, \operatorname{Hom}_{\mathbb{k}}(L(\mathrm{i}), L(\mathrm{j}))\right)$ to the exact sequence $0 \rightarrow \bar{W} \rightarrow W \rightarrow B \rightarrow 0$ yields the long exact sequence

$$
\begin{aligned}
0 \rightarrow \operatorname{Hom}_{B \otimes_{\mathbb{k}} B^{\text {op }}}\left(B, \operatorname{Hom}_{\mathbb{k}}(L(\mathrm{j}), L(\mathrm{i}))\right) & \rightarrow \operatorname{Hom}_{B \otimes_{\mathbb{k}} B^{\mathrm{op}}}\left(W, \operatorname{Hom}_{\mathbb{k}}(L(\mathrm{j}), L(\mathrm{i}))\right) \\
\rightarrow \operatorname{Hom}_{B \otimes_{\mathrm{k}} B^{\mathrm{op}}}\left(\bar{W}, \operatorname{Hom}_{\mathbb{k}}(L(\mathrm{j}), L(\mathrm{i}))\right) & \rightarrow \operatorname{Ext}_{B \otimes_{\mathrm{k}} B^{\mathrm{op}}}^{1}\left(B, \operatorname{Hom}_{\mathbb{k}}(L(\mathrm{j}), L(\mathrm{i}))\right) \\
& \rightarrow \operatorname{Ext}_{B \otimes_{\mathbf{k}} B^{\mathrm{op}}}^{1}\left(W, \operatorname{Hom}_{\mathbb{k}}(L(\mathrm{j}), L(\mathrm{i}))\right) .
\end{aligned}
$$

Using that $\operatorname{Ext}_{B \otimes_{\mathbb{k}} B^{\text {op }}}\left(B, \operatorname{Hom}_{\mathbb{k}}(L(\mathrm{j}, L(\mathrm{i}))) \cong \operatorname{Ext}_{B}^{s}(L(\mathrm{j}), L(\mathrm{i}))\right.$ for $s=0,1$, the fact that

$$
\operatorname{Ext}_{B \otimes_{\mathbb{k}} B^{\text {op }}}\left(W, \operatorname{Hom}_{\mathbb{k}}(L(j), L(\mathrm{i}))\right) \cong \operatorname{Ext}_{\Lambda}^{s}(\Delta(\mathrm{j}), \Delta(\mathrm{i})),
$$

for $s=0,1$, see e.g. [KM19, Section 4], and the fact that $B$ is directed, and therefore $\operatorname{Ext}_{B}^{1}(L(j), L(i))=0$ for $j \geq i$, the long exact sequence 3.13.1 can be rewritten to obtain the exact sequence

$$
\begin{aligned}
0 \longrightarrow \operatorname{Hom}_{B}(L(\mathrm{j}), L(\mathrm{i})) & \longrightarrow \operatorname{Hom}_{\Lambda}(\Delta(\mathrm{j}), \Delta(\mathrm{i})) \\
& \longrightarrow \operatorname{Hom}_{B \otimes_{\mathrm{k}} B^{\mathrm{op}}}\left(\bar{W}, \operatorname{Hom}_{\mathbb{k}}(L(\mathrm{j}), L(\mathrm{i}))\right) \longrightarrow 0 .
\end{aligned}
$$

In particular, a summand $B e_{\mathrm{i}} \otimes_{\mathbb{k}} e_{\mathrm{j}} B$ of $\bar{W}$ gives rise to a non-isomorphism from $\Delta(\mathrm{j})$ to $\Delta(i)$. As $\Lambda$ is quasi-hereditary, this is impossible for $j \geq i$. Regularity follows from Lemma 3.12. The two constructions are inverses to each other by the dual coring theorem [Swe75, 3.7]; see [BW03, 17.11]. 
The following example provides two instances of exact Borel subalgebras which are not homological, respectively homological but not regular.

Example 3.14. (i) As explained in [KKO14, Appendix A.4], in the case of the algebra of Example 2.14, there is an extension between $\Delta(1)$ and $\Delta(3)$ which does not come from an extension of $L_{B}(1)$ and $L_{B}(3)$.

(ii) As explained before, examples of non-regular exact Borel subalgebras correspond to non-regular bocses. An example was given in [KKO14, Appendix A.2] where the quasi-hereditary algebra is $\Lambda=\mathbb{k} \times M_{2}(\mathbb{k})$ and

$$
B=\left\{\left(a,\left(\begin{array}{ll}
a & b \\
0 & c
\end{array}\right)\right) \mid a, b, c \in \mathbb{k}\right\},
$$

which is an exact Borel subalgebra coming from a non-regular (normal) bocs.

\section{REFERENCES}

[AR91] Maurice Auslander and Idun Reiten. On a theorem of E. Green on the dual of the transpose. In Representations of finite-dimensional algebras (Tsukuba, 1990), volume 11 of CMS Conf. Proc., pages 53-65. Amer. Math. Soc., Providence, RI, 1991.

[BPS17] Raymundo Bautista, Efrén Pérez, and Leonardo Salmerón. Tame and wild theorem for the category of filtered by standard modules of a quasi-hereditary algebra. Preprint arXiv:1706.07386.

[BSZ09] Raymundo Bautista, Leonardo Salmerón, and Rita Zuazua. Differential tensor algebras and their module categories, volume 362 of London Mathematical Society Lecture Note Series. Cambridge University Press, Cambridge, 2009. x+452 pp.

[BK18] Agnieszka Bodzenta and Julian Külshammer. Ringel duality as an instance of Koszul duality. Journal of Algebra, 506: 129-187, 2018.

[BB91] William L. Burt and Michael Charles Richard Butler. Almost split sequences for bocses. In Representations of finite-dimensional algebras (Tsukuba 1990), volume 11 of CMS Conference Proceedings, page 89-121. American Mathematical Society, Providence, RI, 1991.

[BW03] Tomasz Brzeziński and Robert Wisbauer. Corings and comodules, volume 309 of London Mathematical Society Lecture Note Series. Cambridge University Press, Cambridge, 2003. xii +476 pp.

[CPS88] Edward Cline, Brian J. Parshall, and Leonard L. Scott. Finite-dimensional algebras and highest weight categories. Journal für die Reine und Angewandte Mathematik. [Crelle's Journal], 391:8599, 1988.

[DR92] Vastimil Dlab and Claus Michael Ringel. The module theoretical approach to quasi-hereditary algebras. In Representations of algebras and related topics (Kyoto, 1990), volume 168 of London Mathematical Society Lecture Note Series, page 200-224. Cambridge University Press, Cambridge, 1992.

[GS17] Edward L.Green and Sibylle Schroll. On quasi-hereditary algebras. Preprint, arXiv: 1710.06674, 2017.

[Kle84] Mark Kleiner. The dual ring to a coring with a grouplike. Proceedings of the American Mathematical Society, (4):540-542, 1984.

[KR77] Mark Kleiner and Andrei Roŭter. Representations of differential graded categories. In Matrix problems (Russian), page 5-70. Akad. Nauk Ukrain. SSR, Inst. Mat., Kiev, 1977.

[KK99] Michael Klucznik and Steffen Koenig. Characteristic tilting modules over quasi-hereditary algebras. Preprint-Server of the SFB 343, E99-004, Universität Bielefeld, 1999.

[Koen95] Steffen Koenig. Exact Borel subalgebras of quasi-hereditary algebras. I. Mathematische Zeitschrift, 220(3):399-426, 1995. With an appendix by Leonard Scott. 
[KKO14] Steffen Koenig, Julian Külshammer, and Sergiy Ovsienko. Quasi-hereditary algebras, exact Borel subalgebras, $A_{\infty}$-categories and boxes. Advances in Mathematics, 262:546-592, 2014.

[KM19] Julian Külshammer and Vanessa Miemietz. Uniqueness of exact Borel subalgebras and bocses. In preparation, 2019.

[Lam99] Tsit-Yuen Lam. Lectures on modules and rings, volume 189 of Graduate Texts in Mathematics. Springer-Verlag, New York, 1999.

[Roi79] Andrei Roĭter. Matrix problems and representations of BOCSes. In Representations and quadratic forms (Russian), 3-38, 154. Akad. Nauk Ukrain. SSR, Inst. Mat., Kiev, 1979.

[Sco87] Leonard L. Scott. Simulating algebraic geometry with algebra. I. The algebraic theory of derived categories. In The Arcate Conference on Representations of Finite Groups (Arcata, Calif., 1986, volume 47 of Proc. Sympos. Pure Math., page 271-281. American Mathematical Society, Providence, RI, 1987.

[Swe75] Moss Sweedler. The predual theorem to the Jacobson-Bourbaki theorem. Transactions of the American Mathematical Society, 213:391-406, 1975.

Tomasz Brzeziński, Department of Mathematics, Swansea University, Swansea UniverSity Bay Campus, Fabian Way, Swansea SA1 8EN, U.K.

Department of Mathematics, University of Bialystok, K. Ciolkowskiego 1M, 15-245 Biąystok, Poland

E-mail address: T.Brzezinski@swansea.ac.uk

Steffen Koenig, Institute of Algebra and Number Theory, University of Stuttgart, Pfaffenwaldring 57, 70569 Stuttgart, Germany

E-mail address: skoenig@mathematik.uni-stuttgart.de

Julian Külshammer, Department of Mathematics, Uppsala University, Box 480, 75106 UPPSAla, SWEDEN

E-mail address: julian.kuelshammer@math.uu.se 